\title{
TILTING BODY VEHICLES ON SLOVAK RAILWAYS - POTENTIAL FOR USE AND PARAMETERS TO BE CONSIDERED
}

Competition between various transport modes has been a characteristic feature of the last decades not only in the world yet in Slovakia. Passenger rail transport proves to be most suitable for medium distances with high traffic intensity. In Slovakia this is valid first of all for Bratislava - Zilina - Kosice line. One solution for increasing travel speed and ride comfort is using the tilting body trains. In the paper theoretical considerations for use of tilting body vehicles are presented. On the example of Zilina - Kosice railway line we show a potential for remarkable reduction of travel time which is the most expected benefit of the new train technology, But we present also parameters which should be considered when thinking about use of new technology.

\section{Introduction}

Competition between various transport modes has been a characteristic feature of the last decades not only in the world yet in Slovakia. Road and air transport are continually growing while railways seem to be declining. However, railway transport is always proclaimed to be a more environmental friendly and more efficient than the other ones, thus giving at least verbal support. But railway transport itself has to prove its benefits showing in reality which parameters are better comparing with other modes as well eliminate its weaknesses. Passenger rail transport proves to be most suitable for medium distances with high traffic intensity. In Slovakia this is valid first of all for Bratislava - Zilina - Kosice line, but other lines, especially connections to the Czech Republic are of interest as well.

In sixties of the $20^{\text {th }}$ century most of leading railways in the world realised that if the railway traffic should withhold in competition with other traffic modes than passenger travel times should have been reduced substantially.

High-speed railway track can not be a universal solution for increasing speed. In a country with high population or mountainous terrain, or where government is not much in favour of investments into a new railway infrastructure, railway management have to find another way for ensuring the competitive travel speed.

As an example of market pressure is that in this situation vehicle designers were challenged to solve somehow the constraints given by conventional technology, geographical and financial conditions.

In leading countries of Europe, North America and Japan these problems were solved by development of a system using tilting vehicle bodies that enabled the train running in curves at a higher speed.

\section{Theoretical background of body tilting and running in curve}

According to the second Newton's law, a body continues in its movement until external force is acting on it. As a consequence of external force action, a body will change its movement velocity and degree of this change is called acceleration.

Passenger trains normally have acceleration/ deceleration up to about $7-9 \%$ gravity force $(9 \% \mathrm{~g})$. This is given by adhesion acting between the wheel and rail.

For acceptable acceleration with respect to passenger comfort the maximum of something less than $10 \% \mathrm{~g}$ is considered.

While instead of acceleration/deceleration on a straight track a train is turning into curve, the Newton law is still valid. Passenger bodies tend to continue in a straight line while the effect of transversal forces accelerates them in a new direction.

This "transversal acceleration" is more perceived by passengers than deceleration or acceleration rate. It occurs very frequently on a line with curves and its magnitude depends on the track shape and train velocity.

Same as acceleration, the transversal acceleration can be expressed in \% g. Normally the transversal acceleration maximum value is $7 \% \mathrm{~g}$, which roughly corresponds to the train braking from speed of $160 \mathrm{~km} / \mathrm{h}$. In Switzerland, the country with railway

\footnotetext{
* Miloslav Klinko ${ }^{1}$, Juraj Grencik ${ }^{2}$

${ }^{1}$ Department for Organization and Management, General-directorate ZSR, Bratislava, Slovakia, E-mail: Klinko.Miloslav@zsr.sk

${ }^{2}$ Department of Transport and Operating Technics, Faculty of Mechanical Engineering, University of Zilina, Slovakia
} 
lines with numerous curves the maximum $9 \% \mathrm{~g}$ is permitted, in France $8.6 \% \mathrm{~g}$, exceptionally, when for example, one curve should remarkably affect the speed, $12 \% \mathrm{~g}$ is accepted.

Civil engineers can compensate the action of transversal acceleration by superelevation of the outer rail - cant of track. During running in a curve the combination (resultant) of transversal force and body mass acts in a direction slanted in a certain angle relative to the vertical. The balance apparatus in the human ear senses this angular tension as turning.

The superelevation was traditionally measured in inches and from Stephenson's era (on BR tracks with a standard rail gauge) one inch $(25 \mathrm{~mm})$ of superelevation corresponds to one degree of slant.

On a railway line with a combined traffic, size of superelevation in a certain curve is always a compromise. Too many slow trains on the lines cause an increased load on the internal rail and resulting increased wear of the wheels and rails, for speed trains the superelevation is limited by passenger comfort.

We should bear in mind that certain transversal acceleration is acceptable and trains are allowed to run in curves at a higher speed than corresponds to the balanced (compensated) acceleration. This uncompensated acceleration is known as cant deficiency.

The maximum speed at which the train is allowed to run in a curve is given by cant deficiency plus maximum superelevation (cant) for freight transport. Danger of overturning caused by transversal forces as a limiting factor need no be considered as this would happen at more than 20 degrees of cant (depending on a vehicle design).

Another important factor is the rate of change of the vehicle tilt as this has great effect on passenger comfort. Tilting effects can be measured in degrees per second. Experimental evaluation of how passengers perceive the tilting showed that at a rate of 5-6 degrees per second they begin perceive the tilting.

Very critical is the acting of tilt in opposite curves when train goes from a maximum superelevation/tilt on one side to a maximum on the opposite side. The British experimental tilting train APT was able to change from the tilt of 9 degrees and cant of 4.5 degree on one side to the similar values on the opposite side within three seconds, which was about 9 degrees per second. This has disturbing effects even in a sitting position. When standing and the rotation axle is in the level of knees, this can easily cause a person to fall down.

What is necessary is to somehow register tilting in advance. A simple and effective solution is placing the accelerometer on the first bogie of a train and to transmit the signals form there along the train with a corresponding delay for individual vehicles in the train. In this way each vehicle receives signal on tilt when it comes to the beginning of a transient curve. This solution is known as a preliminary registration and has also an advantage that it enables reducing a number of processors and thus increases also reliability of the whole system. But the first vehicle still suffers from delay.

Fiat came probably with the best elaborated solution: besides the accelerometer the first bogie was equipped with a sensitive gyroscope and the apparatus for measurement of rail cant.

This gyroscope can register already the beginning of cant on a transient section and transmits the signal to the tilting mechanism. It will start working earlier than the transversal acceleration reaches the value that is registered by the accelerometer.

For trains of series X - 2000 developed by a Swedish company $\mathrm{ABB}$, the solution is partly different: an instantaneous slant angle is measured for each vehicle. When a vehicle enters a section with a transient curve, the tilt angle is continually increasing towards the next vehicle. The signal that registers this difference of tilts is added to the signal transmitted from the accelerometer from the first vehicle. This system improves tilt control and enables more intensive action of the signal from the accelerometer. It is important for prevention of random transversal accelerations, e.g. in a straight track or on imperfect track conditions.

In the era of the first series of APT-P and similar trains problems of passengers were connected with an extremely high compensation of transversal acceleration. Soon it became evident that tilting is too perfect. A standing position or walking is possible thanks to the processing of information in human brains. During sitting in a train with a perfect compensation of transversal acceleration, signals from the inner ear say that the body is in erected position. When one looks out of the window, visual information does not correspond to this information as the train is slanted by $10 \%$. In that moment the brain registers stability problems and in sensitive individuals a phenomenon called nausea occurs, which may cause vomiting.

BR denied that this "nausea from tilt" would be any problem. But other railways found quite a simple answer: instead of the perfect compensation they introduced a tilting system compensating only $70 \%$ of cant deficit, so the remaining transversal acceleration acting on passengers induced a feeling that they are moving in curve. As a consequence, the maximum tilt angle can be reduced and standard of manufacturers stabilised at $8 \%$ - with exception of Pendolino system, in which ETR train is adapted for a tilt of 10 degrees plus $8 \% \mathrm{~g}$ of an uncompensated transversal acceleration - and to $4.8 \%$ cant deficiency.

\section{Calculation of velocity of tilting body train}

For speed trains so called unbalanced acceleration $a_{n}$ is being used, which corresponds to the cant deficiency

$$
a_{n}=\frac{70}{1500} \cdot g \quad \Longrightarrow \quad a_{n}=0.457 \mathrm{~m} \cdot \mathrm{s}^{-2}
$$


The basic formula for calculation of a maximum permitted velocity the following formula is used:

$$
V=\sqrt{\frac{r}{11.8} \cdot\left(p_{m}+p_{n p}\right)}\left(\mathrm{km} \cdot \mathrm{h}^{-1}\right)
$$

where

$r \quad$ - curve radius (m)

$p_{m}$ - maximum superelevation $(\mathrm{mm})$

$p_{n p}$ - cant deficiency (mm)

A further increase of speed without reducing the passenger ride comfort $\left(a_{n}=0,457 \mathrm{~m} \cdot \mathrm{s}^{-2}\right)$ can be gained by tilting the car body inwards toward the curve by a certain angle $\tau$, which represents an additional superelevation $p_{d}$.

$$
p_{d}=e \cdot \operatorname{tg} \tau(\mathrm{mm})
$$

where

$e \quad-$ wheelset/rail contact distance ( $\mathrm{mm})$

We get the permissible velocity with the use of a tilted body

$$
V=\sqrt{\frac{r}{11.8} \cdot\left(p_{m}+p_{n p}+p_{d}\right)}\left(\mathrm{km} \cdot \mathrm{h}^{-1}\right)
$$

This is not a complete solution because the increase of velocity besides balancing the centrifugal force effecting passengers, brings an increase of vehicle effects on the track, too. The vehicle forces acting in a curve on the track, that is safety against derailment and track stability, become a dominant criterion.

Foreign railways use the Proud'hom's formulae when they prepare operation of tilting body vehicles. These formulae give limit of the maximum transversal force between the wheel and rail, which results in an unbalanced centrifugal acceleration of the vehicle $a_{v n}=1.65 \div 1.8 \mathrm{~m} \cdot \mathrm{s}^{-2}$ and only exceptionally $a_{v n}=$ $=2.0 \mathrm{~m} \cdot \mathrm{s}^{-2}$.

A part of the transversal compensated acceleration $a_{v k}$ is eliminated by the rail superelevation $p_{m}$, so the resulting transversal acceleration can be increased by this value. The maximum permissible speed of the tilting body vehicle will then be:

$$
V_{d m}=v_{d m} \cdot 3.6=3.6 \cdot \sqrt{a_{c} \cdot r} \quad(\mathrm{~km} / \mathrm{h})
$$

where

$a_{c}-$ total transversal acceleration $\left(\mathrm{m} . \mathrm{s}^{-2}\right)$

$a_{v k}$ - transversal compensated acceleration $\left(\mathrm{m} . \mathrm{s}^{-2}\right)$

$$
a_{c}=a_{v n}+a_{v k}\left(\mathrm{~m} \cdot \mathrm{s}^{-2}\right)
$$

$a_{v n}$ - uncompensated acceleration according to Proud 'hom's formulae $\left(\mathrm{m} . \mathrm{s}^{-2}\right)$

$$
a_{v k}=\frac{p_{m}}{e} \cdot g\left(\mathrm{~m} . \mathrm{s}^{-2}\right)
$$

$e \quad-$ wheelset/rail contact distance $(\mathrm{mm})$

$r$ - curve diameter $(\mathrm{m})$
Further on we need to determine how a big angle of the body tilt is needed.

$$
a_{c}=a_{v k}+a_{n}+a_{d}\left(\mathrm{~m} \cdot \mathrm{s}^{-2}\right)
$$

where

$a_{n}$ - unbalanced acceleration $\left(\mathrm{m} . \mathrm{s}^{-2}\right)$

$$
a_{d}=a_{c}-a_{v k}-a_{n}\left(\mathrm{~m} \cdot \mathrm{s}^{-2}\right)
$$

$a_{d}$ - additionally compensated acceleration $\left(\mathrm{m} \cdot \mathrm{s}^{-2}\right)$

$p_{d}$ - additional superelevation $(\mathrm{mm})$

From the following formula we get the required angle of a body tilt:

$$
p_{d}=\frac{a_{d}}{g} \cdot e \quad \operatorname{tg} \tau=\frac{p_{d}}{e} \Rightarrow \tau
$$

\section{Computer simulation of a train run}

One of the most important steps in the decision process on the use of tilting body vehicles is also a theoretical calculation of run parameters on the proposed railway line section.

For this calculation we used a program "Train dynamics". Parameters of the selected railway line section have to be input into the program.

A) Input data of the program comprise:

1. Parameters of a selected railway line section

2. Type of locomotive (or traction vehicle)

3. Type of train

4. Complementary data:

- Braking deceleration

- Traction force limit

- Limit of push force

5. Specific data:

- Integration step

- Minimum coasting speed

- Specific heating power

- Coefficient of relative decrease of speed during coasting

- Distance step for data output

B) Results of a sample calculation

After correctly performed calculation we can show the results in the form of a table or diagrams. In the table, besides travel time, the total energy consumption in $\mathrm{kWh}$ or diesel in litres can be presented. For electric locomotive the consumption is given without consumption of auxiliary drives and heating. These items can be considered either by a coefficient or by additional calculation.

The obtained data from the simulation of a train run are certainly only theoretical, but they can provide a starting point for comparison of various types of train sets and testing their para- 
meters from the point of view of their suitability for the selected railway line section.

As an example of the train run simulation using the computer program "Train dynamics" we chose a railway line section Zilina - Kosice, as this section has a considerable number of curves with small radii. (We used the parameter $1 / r, r$ - track curve radius).

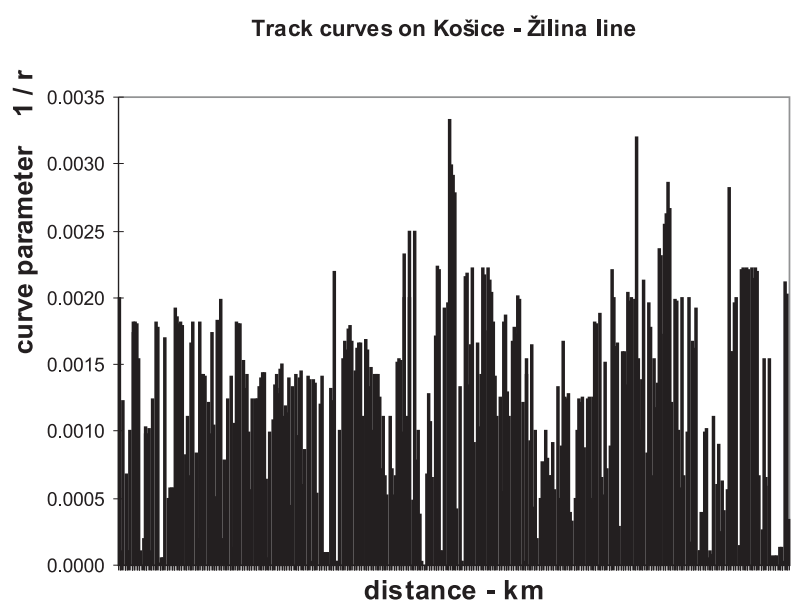

Fig. 1 Track curves on Kosice-Zilina line

We compared the train sets with corresponding weights:

- Conventional - locomotive series 163, plus passenger cars of total weight of $400 \mathrm{t}$, totally $484 \mathrm{t}$.

- With a tilting body - train-set series ETR 470, total weight of $490 \mathrm{t}$, permanent power of $5880 \mathrm{~kW}$, maximum traction force $258 \mathrm{kN}$

Although the power of locomotive series 163 is remarkably lower, for the maximum speed of $120 \mathrm{~km} / \mathrm{h}$ currently used on given railway lines this locomotive is good enough. Calculated travel times for the conventional locomotive-hauled train and Pendolino train set series ETR 470 on the lines with existing speed limits do not differ by more than $1 \%$ in favour of Pendolino, which is a negligible difference. However, benefits from the increased speed in

Table: Travel times and energy consumption on selected sections

\begin{tabular}{|c|c|c|c|c|c|c|}
\hline & \multicolumn{2}{|c|}{$\begin{array}{c}\text { Track sections } \\
\text { existing state }\end{array}$} & \multicolumn{2}{|c|}{$\begin{array}{c}\text { Existing sections } \\
\text { for tilting body } \\
\text { vehicles }\end{array}$} & \multicolumn{2}{|c|}{$\begin{array}{c}\text { Theoretical } \\
\text { sections for } \\
\text { tilting body } \\
\text { vehicles }\end{array}$} \\
\hline $\begin{array}{c}\text { Zilina - } \\
\text { Kosice }\end{array}$ & $\begin{array}{c}\text { Travel } \\
\text { time } \\
(\mathrm{min})\end{array}$ & $\begin{array}{c}\text { Energy } \\
\text { consum } \\
\text { ption } \\
(\mathrm{kWh})\end{array}$ & $\begin{array}{c}\text { Travel } \\
\text { time } \\
(\mathrm{min})\end{array}$ & $\begin{array}{c}\text { Energy } \\
\text { consum } \\
\text { ption } \\
(\mathrm{kWh})\end{array}$ & $\begin{array}{c}\text { Travel } \\
\text { time } \\
(\mathrm{min})\end{array}$ & $\begin{array}{c}\text { Energy } \\
\text { consum } \\
\text { ption } \\
(\mathrm{kWh})\end{array}$ \\
\hline $163+400 \mathrm{t}$ & 154.1 & 2374 & - & - & - & - \\
\hline ETR 470 & 153.6 & 1626 & 110.7 & 1879 & 102.3 & 2776 \\
\hline
\end{tabular}

curves confirmed the results of simplified calculations; although, the difference, according to a more precise calculation, was a bit smaller. Moreover, in these calculations we considered speed limits in the same sections as they are at present, yet we also used theoretical speed limits for each curve while certainly the maximum speed of train ETR 470, which is $200 \mathrm{~km} / \mathrm{h}$, could not be exceeded. Selected calculation results are in the following table.

The diagram of a run diagram and energy consumption on Košice - Zilina line, theoretical sections (considering maximum possible theoretical speed in each curve on the line) is presented in the figure below:

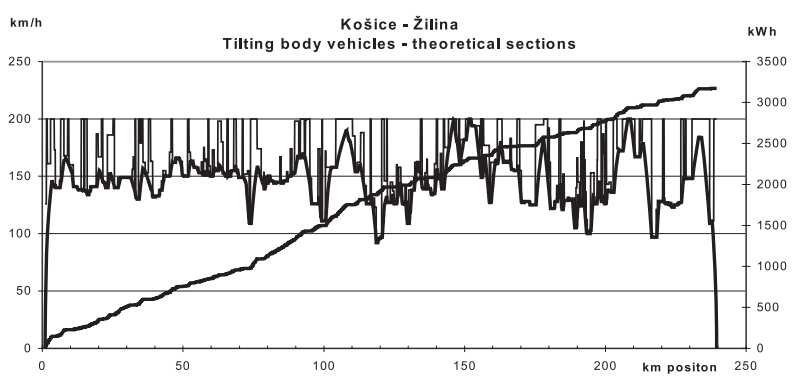

Fig. 2 Tilting body vehicles - theoretical line sections

(Kosice - Zilina line)

In case of theoretical speed limits for each curve increased energy consumption has remarkably gone up, as there were frequent changes in velocity (acceleration in the sections with a higher permissible speed).

\section{Parameters effecting choice of a suitable rail vehicle}

In the decision process on suitability of the use of a selected railway vehicle type on a given railway line it is necessary to investigate not only design parameters of the vehicle but also other parameters concerning its operation have to be considered as well.

These parameters in principle can be divided into the following categories:

a) Technical parameters:

- parameters of railroad tracks and buildings

- parameters of interlocking systems

- parameters of traction power supply and electrotechnical equipment

b) Parameters of effects on environment railroad surroundings

c) Parameters of effect on passengers

d) Economic parameters

- Operational and maintenance costs

- Disposal costs

- Costs for increasing quality and reliability

- Competitiveness with other transport modes 


\section{Technical parameters}

These parameters are based on valid legislation (regulations, standards,...) and mostly in a designing phase of a suitable type of railway vehicle it is not possible to influence these parameters as their change requires high investments for modification of the railway track and other connected technical facilities. Moreover, these parameters directly affect the operational safety of the rail vehicle.

\section{Parameters of effects on environment railroad surroundings}

Parameters of effects on environment railroad surroundings are also given by valid legislation but they can be affected by a vehicle design and its maintenance system. The main parameters belonging to this group are especially:

- Noise emitted by the vehicle;

- Influence on soil, water and air pollution.

\section{Parameters of effect on passengers}

These parameters are most important from the point of meeting passenger needs. They concern namely:
- Personal comfort during the train run

- Speed of transport from place A to place B

- Train ticket price

- Traffic safety

\section{Economic parameters}

Economic parameters are the most important for assessment suitability of the use of a railway vehicle on the given railway line as operation should be economically effective. Individual types of costs related to the rail vehicle operation can be affected already during the design phase.

When considering the individual parameters with the aim of choice of the most suitable type for a given railway line it is good not to focus only on one parameter that looks like the most important, but all the parameters should be evaluated and considered in their mutual connection. This is valid also for usage of tilting body vehicles when one important parameter - an increased train velocity should be considered with all the induced requirements for operation of this train technology.

\section{References}

[1] SIMCO, J: Employment Tilting Body Trains - Alternative for Raising Speed in Railway Transport (in Slovak), Horizonty dopravy 3/2002, Zilina, p. 1-8

[2] SIMAN, P, PETRAS, J.: Programme „SP Dynamika“ (in Czech), Computer program, Praha, 1998

[3] GRENCIK, J., KLINKO, M.: Potential Reduction of Travel Times and Energy Consumption of Tilting Body Vehicles on ZSR, Communications - Scientific Letters of the University of Zilina, 1/2001, EDIS Žilina, p. 88-97. 